\title{
OCORRÊNCIA DE PERDA AUDITIVA INDUZIDA PELO RUÍDO EM CARPINTEIROS
}

\section{Occurrence of noise induced hearing loss in carpenters}

\author{
Victor Hygor Veríssimo Farias ${ }^{(1)}$, Ana Karina Lima Buriti (2), Marine Raquel Diniz da Rosa ${ }^{(3)}$
}

\begin{abstract}
RESUMO
Objetivo: investigar a ocorrência de perda auditiva induzida pelo ruído (PAIR) em carpinteiros, caracterizando a perda auditiva por faixa etária, tempo de exposição total ao ruído e uso regular de protetores auditivos durante o tempo total de exposição. Método: estudo retrospectivo, descritivo, em uma população de 80 carpinteiros da construção civil, atendidos em uma clínica particular. Foram analisados 60 trabalhadores, conforme dados obtidos na anamnese e ficha do exame audiométrico. Resultados: $49 \%$ dos trabalhadores apresentaram audição normal, sendo 58\% com limiares auditivos normais bilateralmente e 35\% com entalhe audiométrico em $3 \mathrm{kHz}, 4 \mathrm{kHz}$ e/ou $6 \mathrm{kHz}$. 44\% apresentaram perfil audiométrico sugestivo de PAIR, destes $74 \%$ foram classificados como PAIR bilateral e $19 \%$ como PAIR unilateral. Houve diferença estatística significante entre os grupo PAIR e Normal em relação às variáveis idade $(p=0,001)$, assim como o tempo total de exposição ao ruído ocupacional $(p=0,002)$. Conclusão: quanto maior a idade e o tempo de profissão como carpinteiro, maior é a sua alteração auditiva, principalmente, devido à exposição ao ruído elevado durante a jornada de trabalho, sendo também constatado que as medidas de controle pelo uso do protetor são insuficientes para prevenir perdas auditivas. Portanto, sugerem-se medidas preventivas em saúde auditiva ativamente nessa população estudada, no ramo da construção civil.
\end{abstract}

DESCRITORES: Ruído; Perda Auditiva Provocada por Ruído; Trabalhadores; Audição

\section{INTRODUÇÃO}

O ruído, termo usado para descrever um som indesejável, é o agente físico nocivo mais comum encontrado no ambiente de trabalho e pode afetar o ser humano nos planos físico, psicológico e social. Devido à alta prevalência de exposição a intensidades sonoras prejudiciais à audição, constitui-se em um importante agravo à saúde dos trabalhadores em todo o mundo ${ }^{1}$.

(1) Fonoaudiólogo; Especialização em Audiologia Clínica pelo Instituto Superior de Ensino de Cajazeiras.

(2) Fonoaudióloga; Especialista em Audiologia Clínica pelo Conselho Federal de Fonoaudiologia; Mestranda do Programa de Pós Graduação em Enfermagem - Universidade Federal da Paraíba.

(3) Fonoaudióloga; Professora Assistente I do curso de Fonoaudiologia Universidade Federal da Paraíba; Doutoranda do Programa de Pós-graduação em Produtos Naturais e Sintéticos Bioativos - Universidade Federal da Paraíba

Conflito de interesses: inexistente
Outros agentes causais de naturezas diversas ao interagir com o ruído podem potencializar seus efeitos sobre a audição, tais como: idade, traumatismo craniano, exposição extraocupacional ao ruído, tabagismo, doenças sistêmicas, história familiar de déficit auditivo e exposição a agentes químicos ocupacionais ${ }^{2,3}$.

As consequências decorrentes da exposição ao ruído podem ser de dois tipos: efeitos na audição e efeitos gerais. No que diz respeito aos efeitos auditivos especificamente, o ruído pode ocasionar três efeitos distintos: o trauma acústico, exposição de forma súbita e intensa que lesa temporária ou definitivamente a audição; a alteração temporária da audição, uma diminuição auditiva reversível; e a perda auditiva induzida por ruído, uma doença crônica caracterizada como uma perda auditiva do tipo neurossensorial, geralmente bilateral, irreversível e progressiva com o tempo de exposição ao ruído ${ }^{4}$.

Além da perda auditiva propriamente dita, o trabalhador exposto ao ruído pode apresentar 
zumbido, hiperacusia, dificuldade de discriminar a fala, como também, alterações do sono e transtornos: da comunicação, neurológicos, vestibulares, digestivos, comportamentais, cardiovasculares e hormonais ${ }^{4-8}$.

A perda auditiva induzida por ruído é o agravo mais frequente à saúde do trabalhador e se encontra presente nos diversos ramos de atividade, dentre eles: siderurgia, metalurgia, gráfica, vidradria, construção civil, entre outros.

Estima-se que o risco de sofrer acidente do trabalho é cerca de duas vezes maior entre trabaIhadores expostos ao ruído. A exposição ocupacional ao ruído não só deteriora a saúde auditiva do trabalhador, mas também se constitui em fator de risco para acidentes do trabalho ${ }^{9}$.

Pesquisas têm demonstrado a prevalência de perda auditiva em trabalhadores expostos ao ruído ${ }^{10,11}$ incluindo metalúrgicos ${ }^{12}$, condutores de ônibus ${ }^{13}$, motoristas de caminhão ${ }^{14}$, marmorarias ${ }^{15}$, dentre outros.

Em se tratando de trabalhadores da construção civil, em que o uso de máquinas cada vez mais velozes tem tornado as tarefas dos trabalhadores mais ruidosas e consequentemente gera perda auditiva e outros efeitos em um número cada vez maior de trabalhadores, ainda são escassas as pesquisas relacionando perda auditiva e construção civil.

A indústria da construção civil é organizada em três níveis hierárquicos: estratégico, intermediário e operacional. Este último constitui-se de mestre de obras, serventes, pedreiros, encarregados, carpinteiros e outros, sendo este o nível da força de trabalho propriamente dita, que executa cotidianamente as tarefas ${ }^{16}$.

Os níveis de pressão sonora das máquinas utilizadas pelos trabalhadores supracitados excedem os níveis de pressão sonora estipulados pela NR-15, Norma Regulamentadora da Portaria MTb n. ${ }^{-}$3.214/1978 ${ }^{17}$, que estabelece os limites de exposição a ruído contínuo. No que diz respeito aos carpinteiros, que fazem parte do madeiramento provisório e permanente da obra, as máquinas por eles utilizadas, tais como ferramentas elétricas e manuais, apresentam níveis de intensidade que variam de 81 a $115 \mathrm{~dB}(\mathrm{~A})$ e frequência de $125 \mathrm{a}$ $16000 \mathrm{~Hz}^{18}$.

A prevalência da perda auditiva na construção civil foi comprovada em algumas pesquisas ${ }^{18,20-22}$, demonstrando perfil audiométrico sugestivo de PAIR e presença de entalhe em trabalhadores com audição normal.

Diante do exposto e tendo em vista que os trabalhadores da Construção Civil são, na sua maioria, pessoas simples que além de enfrentar condições penosas de trabalho não encontram proteção adequada a sua saúde e integridade física, o objetivo desta pesquisa foi investigar a ocorrência de perdas auditivas induzidas pelo ruído em trabalhadores de empresas da Construção Civil, caracterizando as perdas auditivas por faixa etária e tempo de exposição total ao ruído, além de determinar o tempo de uso regular de protetores auditivos durante o tempo total de exposição ao ruído. Buscando assim, dados para a criação de programas de prevenção da perda auditiva nestes trabalhadores em prol da qualidade de vida e de trabalho dos mesmos.

\section{MÉTODO}

Trata-se de um estudo descritivo e retrospectivo. A pesquisa foi realizada em um consultório particular, cujo responsável da clínica consentiu a realização da análise dos prontuários e a divulgação dos dados obtidos para realização de um estudo científico.

Foram cedidos para a realização dessa pesquisa 1119 prontuários de trabalhadores do ramo da construção civil. A seleção da amostra se deu pelo método aleatório de conglomerados, sorteio, composta por grupos de atividades que exerciam a mesma função. Dentre estes, foi sorteado a função de carpinteiro, da qual foram selecionados 80 prontuários de trabalhadores com esta função. Visto que os mesmos estão expostos a ruídos acima de 85 $\mathrm{dB}$ e, partindo do pressuposto que a intensidade do ruído é considerada o fator principal para a perda auditiva ${ }^{19}$, os carpinteiros podem ser incluídos entre os trabalhadores com grande risco de adquirir perda auditiva induzida por ruído.

Para serem incluídos no grupo de estudo, os trabalhadores pertencentes à função de carpinteiro, deveriam atender aos seguintes critérios de inclusão: (1) Função operacional com exposição ao ruído; (2) Possuir os registros audiométricos de exame admissional realizados em 2007 e (3) Anamnese clínica e ocupacional completa.

Assim, dos 80 trabalhadores carpinteiro foram excluídos 20, por não atenderem aos critérios de inclusão. Portanto, a amostra foi composta por 60 trabalhadores do ramo da construção civil expostos a níveis de ruído acima do preconizado na NR 15.

Os exames audiométricos dos 60 trabalhadores foram analisados de acordo com o critério de Mudança Significativa de Limiar ${ }^{23}$, conforme descrito na Figura 1.

A pesquisa foi aprovada pelo Comitê de Ética e Pesquisa da Universidade Federal da Paraíba, sob protocolo 0294/2008, estando de acordo com a Resolução № 196/96. 


\begin{tabular}{|c|c|c|}
\hline \multicolumn{2}{|c|}{ Classificação } & \multirow{2}{*}{ Descrição } \\
\hline Grupo & Subgrupo & \\
\hline Grupo NORMAL & & $\begin{array}{l}\text { Todos os limiares auditivos bilateralmente, iguais } \\
\text { ou menores que } 25 \mathrm{dBNA} \text {. }\end{array}$ \\
\hline Grupo PAIR & & $\begin{array}{l}\text { Audiograma sugestiva de perda auditiva induzida } \\
\text { por ruído, segundo a NR } 15 \text {. }\end{array}$ \\
\hline Grupo OUTROS & & $\begin{array}{l}\text { Exames que não puderam ser classificados como } \\
\text { Normal ou PAIR. }\end{array}$ \\
\hline \multirow[b]{3}{*}{$\begin{array}{l}\text { Grupo } \\
\text { NORMAL }\end{array}$} & Normal Bilateral (NLBI): & $\begin{array}{l}\text { Todos os limiares tanto da orelha direita quanto } \\
\text { da esquerda eram iguais ou inferiores a } 25 \text { dBNA; }\end{array}$ \\
\hline & $\begin{array}{l}\text { Normal com Entalhe Bilateral } \\
\text { (NLEBI): }\end{array}$ & $\begin{array}{l}\text { Idem ao normal bilateral, porém apresentando } \\
\text { entalhe em ambas as orelhas; }\end{array}$ \\
\hline & $\begin{array}{l}\text { Normal com Entalhe } \\
\text { Unilateral (NLEUNI): }\end{array}$ & $\begin{array}{l}\text { Idem ao normal bilateral, porém apresentando } \\
\text { entalhe audiométrico em apenas uma orelha } \\
\text { (rebaixamento dentro da normalidade, nas } \\
\text { freqüências de } 3 \mathrm{kHz}, 4 \mathrm{kHz} \text { ou } 6 \mathrm{kHz} \text {, sendo } \\
\text { considerado quando houver uma diferença de } \\
\text { pelo menos } 10 \mathrm{~dB} \text { da freqüência anterior ou } \\
\text { posterior). }\end{array}$ \\
\hline \multirow{3}{*}{ Grupo PAIR } & PAIR Bilateral (PAIRBI): & $\begin{array}{l}\text { Traçado audiométrico sugestivo de perda auditiva } \\
\text { induzida por ruído em ambas as orelhas; }\end{array}$ \\
\hline & PAIR Unilateral (PAIRUNI): & $\begin{array}{l}\text { Uma orelha com traçado audiométrico sugestivo } \\
\text { de PAIR, com a outra apresentando todos os } \\
\text { limiares normais (iguais ou inferiores a } 25 \text { dBNA); }\end{array}$ \\
\hline & $\begin{array}{l}\text { PAIR Unilateral com Entalhe } \\
\text { na Outra Orelha (PAIRUNIE): }\end{array}$ & $\begin{array}{l}\text { Traçado audiométrico sugestivo de PAIR em uma } \\
\text { orelha e traçado normal com entalhe } \\
\text { audiométrico na outra orelha. }\end{array}$ \\
\hline
\end{tabular}

Figura 1 - Classificação de audiogramas ocupacionais, segundo Fiorini,1994

Os dados obtidos foram submetidos à análise estatística, sendo relacionados entre si. Foi utilizado o programa estatístico SPSS em versão 10 para obtenção dos resultados. Para análise dos dados foi utilizado o Teste Mann-Whitney. Os resultados analisados foram considerados estatisticamente significantes quando $p<0,050$.

\section{RESULTADOS}

O grupo de trabalhadores avaliados foi de 60 trabalhadores da construção civil, que exerciam a função de carpinteiro. O grupo caracterizou-se por predominância absoluta do sexo masculino (100\%), média etária de 39 anos, e desvio padrão de 9,6. A Tabela 1 mostra a distribuição dos trabalhadores pesquisados por faixa etária. Observou-se uma maior concentração na faixa etária de $30-40$ anos $(40 \%)$, seguida pela faixa etária de $40-50$ anos $(26,7 \%)$.

A Tabela 2 apresenta o tempo total de exposição da população pesquisada ao ruído ocupacional, que teve média de 17 anos de exposição e desvio padrão de 9,4. Observou-se um maior número de sujeitos investigados nesse estudo nas três primeiras faixas que vão do 0 a 30 anos de exposição, totalizando 54 indivíduos, isto é, um percentual de $90 \%$ da amostra.

O tempo de uso regular de protetores auditivos durante o tempo total de exposição ao ruído teve uma média de 7 anos com desvio padrão de 6,7 e está melhor representado na Figura 2. Observou-se que mais da metade (63\%) usa os protetores auditivos regularmente há uma faixa de até 10 anos.

A Tabela 3 indica a distribuição dos trabalhadores quanto à classificação dos resultados dos exames audiométricos. Como se pode observar, dos 60 audiogramas estudados, 29 sujeitos (49\%) apresentaram audiogramas dentro dos padrões de normalidade e 31 sujeitos (51\%) apresentam audiogramas com alterações, dessas, $44 \%$ devido a exposições prolongadas ao ruído ocupacional.

A Figura 3 mostra a distribuição dos trabalhadores com audição normal em três subgrupos, conforme figura $1^{20}$. 
Tabela 1 - Distribuição dos trabalhadores por faixa etária

\begin{tabular}{ccc}
\hline FAIXA ETÁRIA & $\mathbf{n}$ & $\%$ \\
\hline $20-30$ & 11 & 18,3 \\
$31-40$ & 24 & 40 \\
$41-50$ & 16 & 26,7 \\
$51-60$ & 8 & 13,3 \\
$61-70$ & 1 & 1,7 \\
\hline TOTAL & $\mathbf{6 0}$ & $\mathbf{1 0 0}$ \\
\hline
\end{tabular}

Legenda: $\mathrm{n}=$ números dos sujeitos.

Tabela 2 - Distribuição da população por tempo total de exposição a ruído ocupacional.

\begin{tabular}{ccc}
\hline TEMPO DE EXPOSIÇÃO AO RUÍDO & $\mathbf{n}$ & $\%$ \\
\hline $0-10$ & 16 & 26,6 \\
$11-20$ & 21 & 35 \\
$20-30$ & 17 & 28,3 \\
$31-40$ & 4 & 7,7 \\
$41-50$ & 2 & 3,3 \\
\hline TOTAL & $\mathbf{6 0}$ & $\mathbf{1 0 0}$ \\
\hline
\end{tabular}

Legenda: $\mathrm{n}=$ números dos sujeitos

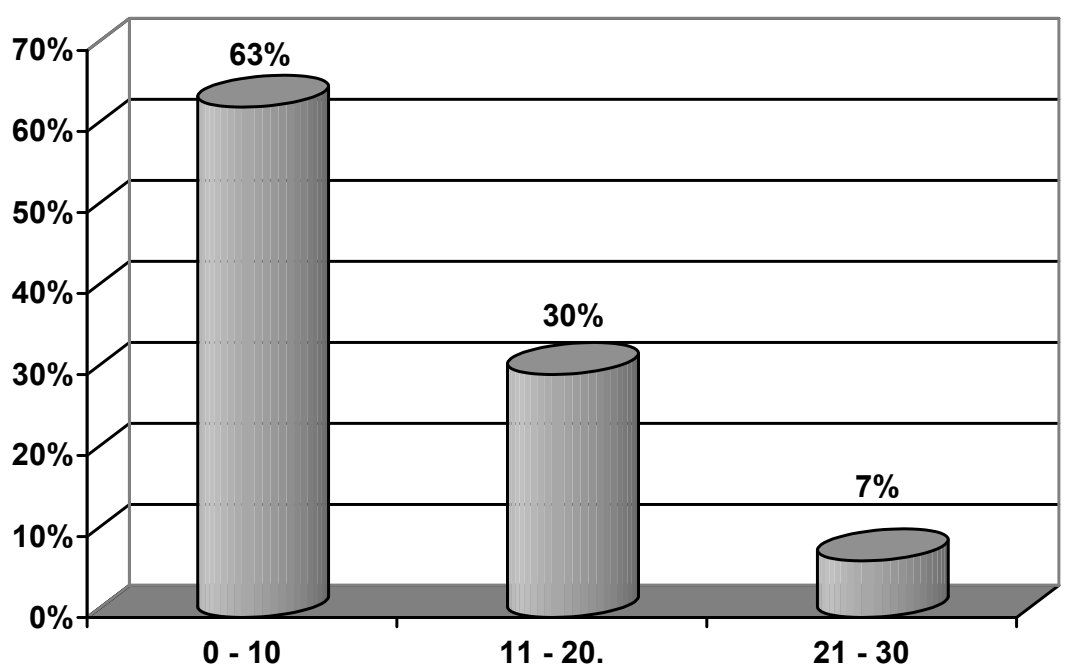

Figura 2 - Distribuição dos trabalhadores de acordo com o tempo (em anos) de uso regular de protetores auditivos

Tabela 3 - Distribuição dos trabalhadores quanto à classificação do exame audiométrico.

\begin{tabular}{ccc}
\hline CLASSIFICAÇÃO DOS RESULTADOS & $\mathbf{n}$ & $\%$ \\
DOS EXAMES AUDIOMÉTRICOS & 29 & $49 \%$ \\
\hline NORMAL & 27 & $44 \%$ \\
PAIR & 4 & $7 \%$ \\
OUTROS & 60 & $100 \%$ \\
\hline TOTAL &
\end{tabular}

Legenda: $\mathrm{n}=$ números dos sujeitos; PAIR = Perda auditiva induzida por ruído. 


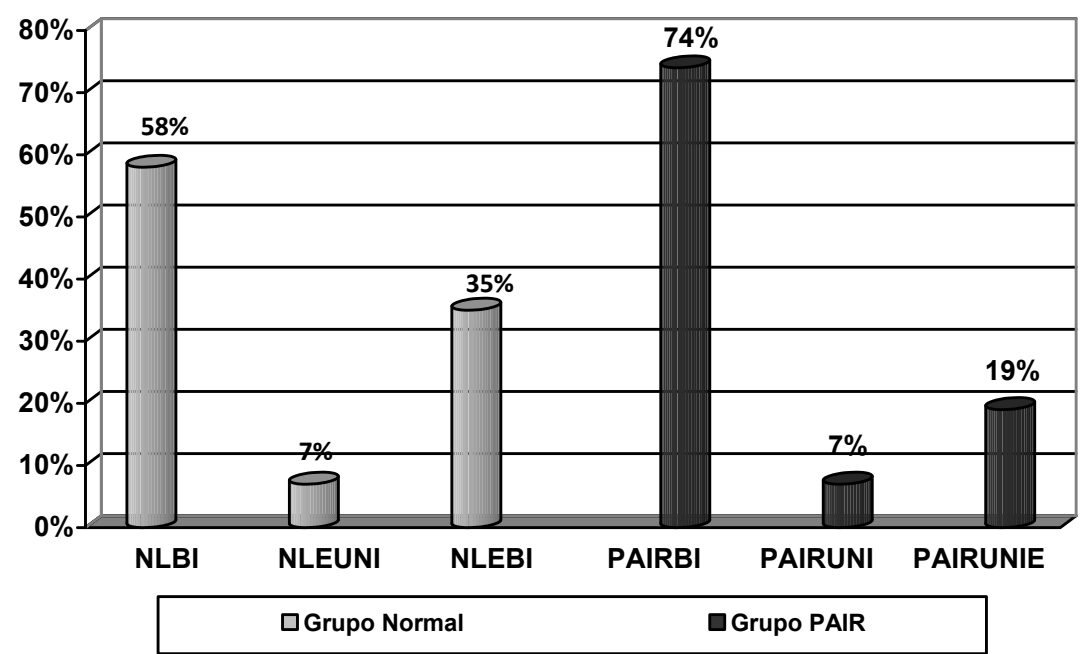

Legenda: NLBI: Normal Bilateral; NLEBI: Normal com Entalhe Bilateral; NLEUNI: Normal com Entalhe Unilateral; PAIRBI: perda auditiva induzida por ruído bilateral; PAIRUNI: perda auditiva induzida por ruído unilateral; PAIRUNIE: perda auditiva induzida por ruído em uma orelha e normal com entalhe na outra.

Figura 3 - Distribuição do grupo Normal e Grupo PAIR em subgrupos

De acordo com o que se observa, há predomínio pelo grupo normal, ou seja, que apresenta audiograma dentro dos padrões de normalidade, sem entalhe $(58 \%)$, seguidos por trabalhadores com audiograma dentro dos padrões de normalidade e entalhe bilateral (35\%) e de trabalhadores com audiograma dentro dos padrões de normalidade apenas unilateral, estando o outro lado com entalhe $(7 \%)$.

Na Figura 3, observa-se também o grupo PAIR subdividido em três subgrupos. Dos 27 audiogramas alterados deste grupo, 20 (74\%) apresentaram classificação sugestiva de PAIR bilateral, dois (7\%) eram sugestivos de PAIR unilateral com audição dentro dos padrões de normalidade na orelha contralateral, sem entalhe e cinco (19\%) eram classificados como sugestivo de PAIR unilateral, sendo que a orelha contralateral apresentou audição dentro dos padrões de normalidade, mas com entalhe.

Dentre os 60 trabalhadores analisados, apenas quatro $(7 \%)$ tiveram seus resultados classificados no grupo de exames que não puderam ser classificados como Normal ou PAIR (Grupo OUTROS).

Visando-se verificar a influência das variáveis idade, tempo de exposição ao ruído e tempo de uso regular de protetores auditivos nos casos sugestivos de PAIR realizou-se o teste de regressão logística e comparação de seus resultados por meio do teste de Mann-Whitney, o que pode ser melhor visualizado na Tabela 4.

A Tabela 4 mostra que os trabalhadores do Grupo PAIR apresentam maior tempo médio de exposição ao ruído ocupacional e são mais velhos quando comparados aos do Grupo NORMAL.

Tabela 4 - Distribuição das variáveis: tempo total de exposição ao ruído ocupacional, tempo de uso regular de protetores auditivos e idade, considerando os resultados obtidos na avaliação audiométrica dos carpinteiros, por meio da categorização em grupos proposta por Fiorini (1994) $(n=56)$ e sua comparação pelo Teste de Mann-Whitney

\begin{tabular}{|c|c|c|c|c|}
\hline TEMPO (ANOS) & MÉDIA_DP & GRUPO PAIR & GRUPO NORMAL & $\begin{array}{c}\text { VALOR-P } \\
\text { MANN-WHITNEY }\end{array}$ \\
\hline $\begin{array}{l}\text { Exposição ao } \\
\text { ruído ocupacional }\end{array}$ & $16,88 \pm 9,46$ & $21,11 \pm 9,56$ & $13,19 \pm 7,78$ & $0,002^{*}$ \\
\hline $\begin{array}{l}\text { Uso regular do } \\
\text { protetor auditivo }\end{array}$ & $7,62 \pm 6,69$ & $6,61 \pm 5,71$ & $8,50 \pm 7,41$ & 0,409 \\
\hline Idade & $38,83 \pm 9,60$ & $43,36 \pm 9,08$ & $34,88 \pm 8,32$ & $0,001^{*}$ \\
\hline
\end{tabular}

Legenda: ${ }^{*}<<0,05$ 


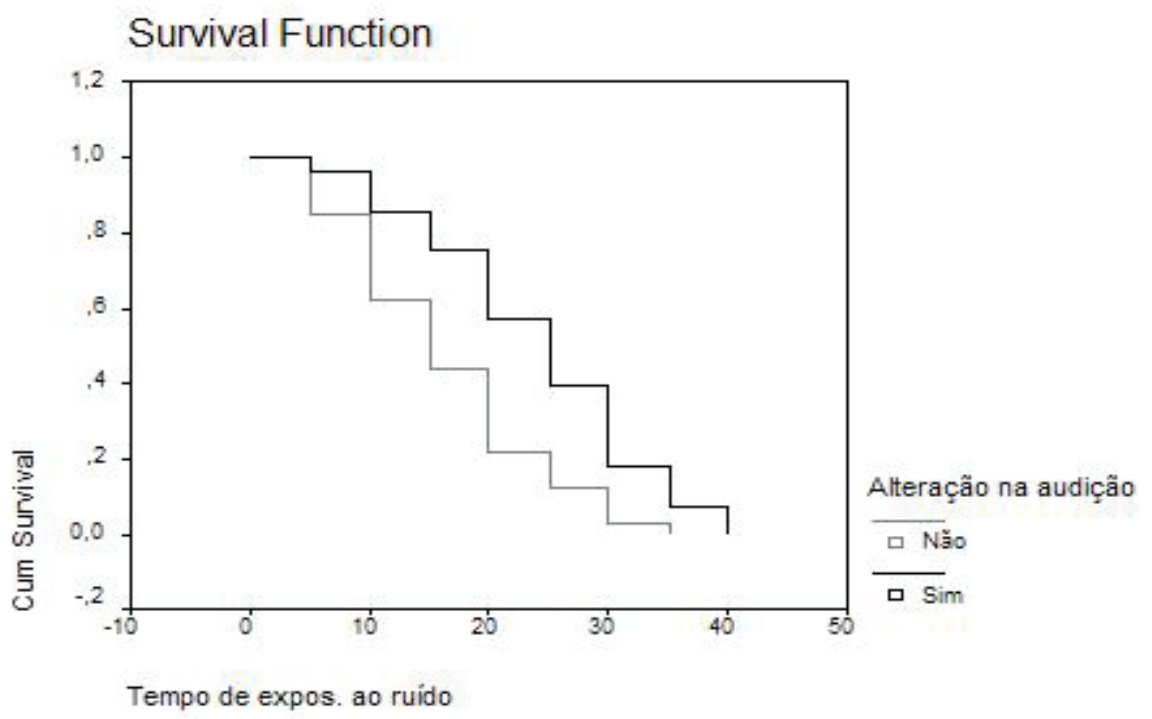

Figura 4 - Curva de sobrevivência para o tempo total de exposição ao ruído

Segundo o teste de Mann-Whitney, há evidência estatística $(p<0,05)$ de que estas diferenças são significantes. Não há evidência estatística segundo este mesmo teste de que o tempo regular de uso de proteção auditiva seja diferente nos dois grupos de trabalhadores.

A variável tempo de exposição foi demonstrada através da análise de sobrevivência apresentada na Figura 4, onde o evento bem definido (falha) ocorre depois de algum tempo. De acordo com a análise, os indivíduos sob risco não falharam e não foram censurados até o instante.

A Figura 4 mostra que o grupo de trabalhadores com alteração auditiva sugestiva de PAIR apresenta uma curva com valores sempre maiores do que os do grupo Normal. Portanto, fica evidenciado o fato de que o grupo de trabalhadores com alteração auditiva tem um histórico de tempo de exposição ao ruído superior ao outro grupo.

\section{DISCUSSÃO}

Sabe-se que o ruído constitui um sério problema para a sociedade moderna, que afeta a audição e também outras esferas da saúde física e mental.

Um dado alarmante encontrado neste estudo foi a ocorrência de perdas auditivas sugestivas de PAIR encontrada nesta classe profissional de trabalhadores expostos a ruído. Comparando-se os dados desta pesquisa com outros estudos na literatura ${ }^{12,15,24}$, observa-se que há uma grande ocorrência de perdas auditivas sugestivas de PAIR (44\%), corroborando assim com os $15,9 \%$ dos trabaIhadores de metalúrgicas, $50 \%$ dos trabalhadores de mamorarias e $12,71 \%$ dos trabalhadores de diversos ramos industriais (metalúrgico, tecelagem, calçadista, químico, plástico, siderúrgico, couro, produtos minerais, alimentício, bebidas, celulose, eletroeletrônico, madereiro, confecção e outras) de um Distrito.

Um estudo anterior envolvendo carpinteiros ${ }^{20}$ mostrou resultados semelhantes a esta pesquisa, pois encontrou que pouco mais de $51 \%$ dos trabaIhadores apresentaram curvas audiométricas sugestivas de PAIR.

Neste estudo, na tabela 3 , observou-se que 29 trabalhadores (49\%) apresentaram audição normal. No entanto, foi evidenciado na Figura 3, que no grupo normal 17 trabalhadores (58\%) apresentaram limiares auditivos normais bilateralmente sem entalhe em ambas as orelhas; 10 (35\%) apresentaram entalhe audiométrico em $3 \mathrm{kHz}, 4 \mathrm{kHz}$ e/ ou $6 \mathrm{kHz}$, ainda que todos os outros limiares bilateralmente estivessem iguais ou menores a $25 \mathrm{~dB}$ $\mathrm{NA}$, sugerindo, traços de alteração de estruturas da cóclea por exposição a ruído elevado.

Nesse contexto, a literatura refere que a PAIR inicia-se na faixa de frequência entre 3000 e $6000 \mathrm{~Hz}{ }^{15}$, sendo a frequência de $6000 \mathrm{~Hz}$ a mais acometida ${ }^{13}$. Outro trabalho, entretanto, referiu que a frequência de $4000 \mathrm{~Hz}$ é a mais comprometida nos estágios iniciais ${ }^{4}$.

Em estudo semelhante ${ }^{21}$, que teve como objetivo levantar o perfil audiométrico de 192 trabalhadores da construção civil foram encontradas 14,6\% de curvas audiométricas sugestivas de PAIR, percentual menor em comparação com os percentuais encontrados na população pesquisada neste 
estudo. Quanto à ocorrência de curvas audiométricas com entalhe, apesar de seus limiares encontrarem-se dentro dos padrões de normalidade, o estudo detectou-as em $55,2 \%$, número maior do que o encontrado em nosso estudo, que equivale a $35 \%$.

Em vista desses resultados, enfatiza-se a importância da realização de programas preventivos e de conscientização do trabalhador quanto aos riscos que o ruído produz não só para sua audição, mas também para sua saúde em geral.

Destaca-se a importância de investimentos em programa de conservação auditivas em razão da incidência de acidentes do trabalho decorrente da exposição ao ruído intenso. Os autores sugerem não somente o controle da saúde auditiva, mas atitudes para o controle do ruído na fonte, evitando acidentes de trabalho, como a perda auditiva ${ }^{25}$.

No que diz respeito ao grupo PAIR, pode-se observar na Figura 3 que, dos 27 trabalhadores (44\%) que apresentaram perfil audiométrico sugestivo de PAIR, 20 trabalhadores (74\%) foram classificados no subgrupo PAIRBI, dois (7\%) no subgrupo PAIRUNI e cinco (19\%) no subgrupo PAIRUNIE. Estes resultados se assemelham aos obtidos em outra pesquisa ${ }^{19}$, que encontrou um percentual de $51 \%$ de carpinteiros com audiogramas sugestivos de PAIR, sendo $71 \%$ enquadrados no subgrupo PAIRBI, $16 \%$ no PAIRUNI e $13 \%$ no PAIRUNIE.

Entretanto, outros estudos, 26 envolvendo diversas funções de trabalhadores expostos a ruído em intensidades superiores a $85 \mathrm{dBA}$, referem índice inferior ao encontrado nesta população, detectando apenas $23,5 \%$ de perda auditiva induzida pelo ruído.

A Tabela 4 revelou que há uma diferença estatística entre os dois grupos com relação à idade e tempo de exposição, ou seja, o grupo PAIR tem idade e tempo de exposição ao ruído maior que o grupo normal. Portanto, estes dados corroboraram com a literatura ${ }^{12,27}$, já que, além da suscetibilidade auditiva individual, os carpinteiros poderiam apresentar perdas auditivas em decorrência da idade, associada à exposição constante a níveis de pressão sonora elevados durante sua jornada de trabalho e, além disso, esta exposição ao longo dos anos se caracteriza como um risco potencial para o agravamento ou desencadeamento de PAIR ${ }^{20}$. Assim, afirmam outros autores ${ }^{26}$ que encontraram dados estatisticamente significante entre o tempo de exposição ao ruído e os limiares auditivos para ambas as orelhas, tanto nas altas como nas baixas freqüências.

A literatura relaciona o desenvolvimento da PAIR aos sintomas auditivos e ao tempo de serviço em trabalhadores de diversos ramos industriais, incluindo os da construção civil. Em um estudo foi detectada uma forte associação entre a queixa de hipoacusia e as alterações encontradas principalmente nas frequências altas $(3000,4000$ e $6000 \mathrm{~Hz}$ ). No mesmo estudo verificou-se também que $70 \%$ da população estudada, mesmo com os limiares de 500, 1000 e $2000 \mathrm{~Hz}$ dentro dos padrões de normalidade, apresentaram queixa de hipoacusia. Os autores ressaltaram que esta faixa de frequência é a mais importantes para a comunicação ${ }^{28}$. No entanto, sabe-se que para se perceber essa hipoacusia é necessário que a perda auditiva do indivíduo esteja atingindo a área da fala, ou seja, quando a pessoa começa a referir dificuldades para entender palavras em uma conversação ou mesmo ao telefone.

Neste sentido, a PAIR é considerada como uma doença passível de prevenção, uma vez que o risco ruído pode ser eliminado ou controlado no ambiente de trabalho. Alguns autores ${ }^{26}$ enfatizam a importância da utilização adequada do equipamento de proteção auditiva individual como forma de prevenção de perdas auditivas e/ou promoção da saúde auditiva.Neste estudo, não foi observada diferença estatística do tempo de uso regular de protetor auditivo (PA) no grupo PAIR em relação ao grupo Normal $(p=0,409)$.Tal achado poderia ser explicado pelo fato de que essa variável, que é relatada pelo trabalhador, está muito sujeita a viés de informação, podendo ser super ou subestimada em função de interesses diversos, como por exemplo a obtenção de algum amparo legal. Esse dado corrobora com os achados de pesquisa ${ }^{29}$ realizada com componentes de banda instrumental em que tanto entre os indivíduos com perda auditiva comprovada por meio de avaliação audiológica, como entre aqueles com sintomas auditivos o protetor auditivo foi pouco utilizado.

Ainda pode-se inferir que, possivelmente, as medidas de controle de ruído que vem sendo aplicadas nas tarefas executadas por carpinteiros são insuficientes para prevenir perdas auditivas significantes causadas pelo ruído. É bom ressaltar que, neste estudo, embora a média de tempo total de exposição ao ruído ocupacional seja elevada $(16,88$ anos), a média de tempo de uso regular do protetor auditivo dessa população foi bem menor $(7,62$ anos), quase a metade do tempo.

Pesquisas ${ }^{30}$ demonstram que protetores auditivos tipo plug, os mais utilizados pelos sujeitos da nossa pesquisa, representam uma medida eficaz para atenuação dos níveis de pressão sonora em ambientes ruidosos. E que todos os trabalhadores devem ser devidamente treinados, para que desta forma, utilizando protetores auditivos adequados e bem adaptados, ocorra a atenuação do ruído no 
ambiente de trabalhado, prevenindo assim a perda auditiva induzida por ruído.

O presente estudo possibilitou uma melhor compreensão do comportamento de algumas das principais características relacionadas à PAIR, numa situação particular de organização do trabalho, relativamente comum na construção civil. Certamente, a execução de estudos similares permitirá, cada vez mais, uma interpretação mais próxima da realidade do trabalhador brasileiro, sendo que cada abordagem a ser efetuada deve ser sempre contextualizada, em função da diversidade da saúde ocupacional no Brasil e das dificuldades de se pesquisar tal área no país, que envolvem não somente vários problemas de ordem técnica, mas também interesses financeiros e importantes implicações legais.

Sendo assim, este estudo demonstra a necessidade da implantação de um Programa de Prevenção de Perdas Auditivas (PPPA) em empresas de construção civil, a fim de garantir o monitoramento auditivo, bem como, o treinamento sobre a saúde auditiva dessa classe profissional.

\section{CONCLUSÃO}

Ao final desse estudo pode-se concluir que a audição dos trabalhadores carpinteiros encontra-se alterada, constatando PAIR em $47 \%$ dos trabalhadores analisados, sendo a idade e o tempo de exposição ao ruído (anos de profissão) variáveis que influenciaram significativamente nos resultados da audiometria tonal. Quanto maior a idade e o tempo de profissão como carpinteiro, maior é a sua alteração auditiva, principalmente devido à exposição ao ruído elevado durante a jornada de trabalho. Isto demonstrou o quanto o ruído no ambiente de trabalho pode ser prejudicial para a saúde auditiva destes trabalhadores.

O tempo de uso regular de protetor auditivo apresentou-se irrelevante diante dos casos sugestivos de PAIR. Logo, infere-se que, possivelmente no local onde este estudo foi desenvolvido, as medidas preventivas individuais de controle ao ruído que vem sendo aplicadas nas tarefas executadas por carpinteiros são insuficientes para prevenir perdas auditivas.

\section{ABSTRACT \\ Purpose: to investigate the occurrence of noise induced hearing loss in carpenters, characterizing the hearing loss for age group, time of total exposure to noise and regular use of hearing protectors during the total exposure time. Method: retrospective and descriptive study in a population of 80 construction carpenters, attended at a private clinic. 60 workers were analyzed, as data on medical history and record of audiometric testing. Results: $49 \%$ of the workers shoed normal hearing, being $58 \%$ with normal bilaterally hearing thresholds and $35 \%$ with audiometric notch in $3 \mathrm{kHz}, 4 \mathrm{kHz}$ and/or $6 \mathrm{kHz}$. $44 \%$ showed suggestive audiometric profile of $\mathrm{NIHL}$, of these $74 \%$ were classified as bilateral $\mathrm{NIHL}$ and $19 \%$ as unilateral $\mathrm{NIHL}$. There were significant differences between the NIHL group and normal to the age variables $(p=0.001)$, as well as the total time of exposure to occupational noise $(p=0,002)$. Conclusion: the greater the age and length of employment as a carpenter were, the greater their hearing loss, mainly due to exposure to loud noise during the working day, and we also noted that measures taken in order to control the use of the auricular protector are insufficient to prevent hearing loss. Therefore, we suggest active preventive measures in hearing health in this studied population, in the field of civil construction.}

KEYWORDS: Noise; Hearing Loss, Noise-Induced; Workers; Hearing 


\section{REFERÊNCIAS}

1. Dias A, Cordeiro R, Corrente JE, Gonçalves CGO. Associação entre perda auditiva induzida pelo ruído e zumbidos. Cadernos de Saúde Pública, Rio de Janeiro, 2006;1 (22)63-8.

2. Oliveira TMT, Reis ABF, Bossi C, Pagnossim DF, Pereira, LF, Mello MC et al. Implantação de um programa de conservação auditiva em uma indústria de bebidas. Rev Bras Saude Ocup. 1997;24(89/90):31-6.

3. Morata TC, Lemasters GK. Considerações epidemiológicas para o estudo de perdas auditivas ocupacionais. In: Nudelmann AA, Costa EA, Seligman J, Ibagñez RN. PAIR: perda auditiva induzida pelo ruído. Rio de Janeiro: Revinter. 2001; 2:1-16.

4. Araújo SA. Perda auditiva induzida pelo ruído em trabalhadores de metalúrgica. Rev Bras Otorrinolaringol. 2002; 68(1):47-52.

5. Gonçalves CGO, Iguti AM. Análise de programas de preservação da audição em quatro indústrias metalúrgicas de Piracicaba. Cad Saúde Pública. 2006; 22(3):609-18.

6. Marques FP, Costa EA. Exposição ao ruído ocupacional: alterações no exame de emissões otoacústicas. Rev Bras Otorrinolaringol. 2006; 72(3):362-6.

7. Gonçalves MS, Tocheto TM, Gambini C. Hiperacusia em músicos de banda militar Rev Soc Bras Fonoaudiol. 2007; 12(4):298-303.

8. Gonçalves CGO; Lacerda ABM, Zocoli AMF, Oliva FC, Almeida SB, lantas MR. Percepção e o impacto da música na audição de integrantes de banda militar. Rev Soc Bras Fonoaudiol. 2009; 14 (4): 515-20.

9. Dias A; Cordeiro R; Gonçalves CGO. Exposição ocupacional ao ruído e acidentes do trabalho. Cad Saúde Pública, Rio de Janeiro, 2006;22(10):2125-30. 10. Leão RN, Dias FAM. Perfil audiométrico de indivíduos expostos ao ruído atendidos no núcleo de saúde ocupacional de um hospital do município de Montes Carlos. Rev CEFAC. 2010; 12(2):242-9.

11. Silva AP, Costa EA, Rodrigues SMM, Souza HLR, Massafera VG. Avaliação do perfil auditivo de militares de um quartel do exército Brasileiro. Rev Bras Otorrinolaringol. 2004; 70(3):344-50.

12. Guerra MR, Lourenço PMC, BustamanteTeixeira MT, Alves MJM. Prevalência de perda auditiva induzida por ruído em empresa metalúrgica. Rev. Saúde Pública [online]. 2005; 39(2):238-44.

13. Corrêa Filho HR, Costa LS, Hoehne, EL, Pérez MAG, Nascimento LCR, Moura, EC. Perda auditiva induzida por ruído e hipertensão em condutores de ônibus. Rev. Saúde Pública [online]. 2002; 36(6):693-701.
14. Lopes G, Russo ICP, Fiorini AC. Estudo da audição e da qualidade de vida em motorista de caminhão. Rev CEFAC. 2007; 9(4):532-42.

15. Harger MRHC, Barbosa-Branco A. Efeitos Auditivos decorrentes da exposição ocupacional ao ruído em trabalhadores de marmorarias no Distrito Federal. Rev Assoc Med Bras. 2004; 50(4): 396-9. 16. Leal CMS, Medeiros JADM, Valença SL. Formação de mão de obra de nível operacional na indústria da construção civil. In: Anais IV congresso nacional sobre condições e meio ambiente do trabalho na indústria da construção. Abril 24-27; Goiás (BR) Goiana; FUNDACENTRO. Distrito Federal, 2001.

17. Brasil. Ministério do Trabalho. Portaria $\mathrm{n}^{\circ}$ 3.214, de 8 de Junho de 1978. Aprova as Normas Regulamentadoras - NR - do Capítulo V, Título II, da Consolidação das Leis do Trabalho, relativas a Segurança e Medicina do Trabalho. Diário Oficial da União, Poder Executivo, Brasília, DF, jun. 1978. 18. Maia PA O ruído nas obras da construção civil e risco de surdez ocupacional. [Dissertação]. São Paulo: Universidade Estadual de Campinas Faculdade de Engenharia Civil, 1999. p 153.

19. Boger ME, Barbosa-Branco A, Ottoni AC. The noise spectrum influence on Noise-Induced Hearing Loss prevalence in workers. Braz J Otorhinolaryngol. 2009;75(3):328-34.

20. Roberte R. Estudo longitudinal da audição de carpinteiros da construção civil do estado do Espírito Santo [dissertação de mestrado]. São Paulo: PUC, 2005.

21. Menslin SL. Efeitos do ruído em trabalhadores de uma indústria de construção civil do município de São Paulo [dissertação de mestrado]. São Paulo: PUC, 2001.

22. Martinucci T. Estudo retrospectivo do perfil audiométrico de candidatos a emprego na indústria da construção civil [monografia] curso de especialização do CEDIAU. São Paulo: Campinas, 2002.

23. Fiorini AC. A Importância do Monitoramento Audiométrico no Programa de Conservação Auditiva. São Paulo: Revista Acústica e Vibrações. 1994;13:95-101.

24. Teles RM, Medeiros MPH. Perfil audiométrico de trabalhadores do distrito industrial de Maracanaú - CE. Rev Soc Bras Fonoaudiol. 2007;12(3):233-9. 25. Cordeiro R, Clemente APG, Diniz CS, Dias A. Exposição ao ruído ocupacional como fator de risco para acidentes do trabalho. Rev Saúde Pública. 2005;39(3):461-6.

26. Lopes AC, Nelli MP, Lauris JRP, Amorim RB, Melo ADP. Condições de Saúde Auditiva no Trabalho: Investigação dos Efeitos Auditivos em Trabalhadores Expostos ao Ruído Ocupacional. Arq. 
Int. Otorrinolaringol. / Intl. Arch. Otorhinolaryngol. São Paulo. 2009;13(1):49-54.

27. Bauer $P$, Körpert $K$, Neuberger $M$, Raber A, Schwetz F. Risk factors for hearing loss at different frequencies in a population of 47,388 noise-exposed workers. J Acoust Soc Am. 1991;90(6):3086-98.

28. Ogido R, Costa EA, Machado HC. Prevalência de sintomas auditivos e vestibulares em trabalhadores expostos a ruído ocupacional. Rev Saúde Pública. 2009;43(2):377-80.
29. Mendes MH, Morata TC, Marques JM. Aceitação de protetores auditivos pelos componentes de banda instrumental e vocal. Rev Bras Otorrinolaringol. 2007;73(6):785-92.

30. Faria CAR de, Suzuki FA. Avaliação dos limiares auditivos com e sem equipamento de proteção individual. Rev Bras Otorrinolaringol. 2008;74(3):417-22.

http://dx.doi.org/10.1590/S1516-18462011005000119

RECEBIDO EM: 06/11/2010

ACEITO EM: 23/04/2011

Endereço para correspondência:

Ana Buriti

Rua Severino Massa Spinelli, 200, apto 1701,

Tambaú - João Pessoa - Paraíba

CEP: 58039-210

E-mail: anak_buriti@yahoo.com.br

Rev. CEFAC. 2012 Mai-Jun; 14(3):413-422 